\title{
ELECTRON BEAM AND RF CHARACTERIZATION OF A HIGH- BRIGHTNESS X-BAND PHOTOINJECTOR
}

\author{
D. J. Gibson, F.V. Hartemann, E. C. Landahl, H. A. Baldis, LLNL, Livermore, CA 94550, USA \\ C. H. Ho, Synchrotron Radiation Research Center, Hsinshu 30077, Taiwan \\ A. L. Troha, N. C. Luhmann, Jr. University of California, Davis, CA 95616, USA
}

\begin{abstract}
We report detailed experimental results on an X-band $(8.547 \mathrm{GHz})$ rf photoinjector, currently being commissioned at UC Davis on the LLNL site. The device operates with an average accelerating gradient in the 50$100 \mathrm{MeV} / \mathrm{m}$ range, and produces relativistic $(1.5-2 \mathrm{MeV})$ photoelectron bunches, with a measured relative energy spread $<1.8 \%$, at the resolution limit of our energy spectrometer. The measured quantum efficiency is $2 \times 10^{-5}$, at the maximum of the Schottky curve, and the $90 \%$ normalized emittance is measured at $1.65 \pi-\mathrm{mm} \mathrm{mrad}$ for $20 \mathrm{pC}$ of charge. The bunch duration is extremely short, under the 700 fs resolution of our streak camera. Timing jitter has also been measured directly from the delay between the UV light diffused by the photocathode, and Čerenkov radiation produced by the photoelectrons; a systematic delay is found, corresponding to the time-offlight difference between the UV photons and the accelerated electrons, and the additional timing jitter is estimated at $5 \mathrm{ps}$, or 15 degrees of rf phase. Finally, coherent synchrotron experiments are underway, as the photoelectron bunches radiate coherently up to the $\mathrm{THz}$ region.
\end{abstract}

\section{INTRODUCTION}

Compact, high-gradient $(>100 \mathrm{MeV} / \mathrm{m}) \quad$ rf photoinjectors capable of producing relativistic $(>5 \mathrm{MeV})$ electron beams with extremely low emittance $(<1 \pi$-mm $\mathrm{mrad})$, high charge $(1 \mathrm{nC})$, and subpicosecond duration, are currently being developed for a variety of applications ranging from the Next Linear Collider (NLC) to compact, pre-bunched free-electron masers. Such subcompact electron accelerators will also have a number of important applications in biomedical imaging and therapy, and in drug manufacturing. Finally, these high-gradient electron accelerators will also be extremely useful as basic research tools in universities, industry, and government laboratories.

Within this context, we are developing an X-band $(8.547 \mathrm{GHz})$ rf gun, based on the 1-1/2 cell, S-band Brookhaven National Laboratory (BNL) design[1]. A detailed theoretical and computational study of the scaling of $\mathrm{rf}$ injector beam performance with frequency has been performed[2], with the conclusion that X-band represents an optimum balance between beam quality and accelerated charge and should reach very high brightness. These higher frequency rf photoinjectors offer the possibility of operating at larger peak accelerating fields, thus alleviating the detrimental effects of space charge, which scale as $\gamma^{-1}$, where the identity $\gamma-1=e \boldsymbol{V} / m_{0} c^{2}$ defines the relativistic mass factor, $\gamma$, of the electrons at the potential $\boldsymbol{v}$.

\section{EXPERIMENTAL SET-UP}

\subsection{X-Band RF System}

A thorough description of this system is given elsewhere[3], only a brief summary is presented here. To ensure proper synchronization between the laser and $\mathrm{rf}$ systems, two main options exist: one can either use an external rf clock, which then drives both the high-power rf system energizing the rf gun and the mode-locked laser oscillator, or the rf signal can be derived from the light pulse-train produced by a self-mode-locked laser oscillator. Because of its considerably simpler implementation, the latter option was used in these experiments. A photodiode monitoring the $79.138 \mathrm{MHz}$ oscillator pulse train sends this signal, after filtering, to a phase-locked dielectric resonance oscillator (PDRO), which multiplies the frequency by a factor of 108 , producing a $8.547 \mathrm{GHz}$ signal. The PDRO has an input frequency range of $\pm 50 \mathrm{MHz}$, yielding the frequency tuning required for proper conditioning of the high-power rf gun. This signal is then sent to a $1 \mathrm{~kW}$ travelling wave tube amplifier (TWTA), followed by a Stanford Linear Accelerator Center (SLAC) SL-3 Klystron capable of producing up to $19 \mathrm{MW}$ of rf power in 1-2 $\mu$ s square pulses, at up to a $50 \mathrm{~Hz}$ repetition rate. The power is measured by using a precision thermistor head for the average power, and a fast $(<600 \mathrm{ps}$ rise-time $) \mathrm{rf}$ diode for determination of the pulse duration. For the present experiments, the klystron runs at $20 \mathrm{~Hz}$ with a $250 \mathrm{~ns} \mathrm{rf}$ pulse, to allow for higher gradients in the gun without rf breakdown problems.

The heart of the rf system is a high- $Q(4,274$ measured), $\mathrm{X}$-Band $\mathrm{rf}$ gun. The $\pi$-mode magnetic coupling scheme employed in this design is quite sensitive to mechanical tolerances, in the present case, a custom tuning post piece was fabricated by SLAC to ensure that the peak of the gun resonance is properly correlated with the maximum of the SL-3 rf power tuning curve. This tuning of the rf gun coupling is supplemented by two independent tuners for the half and full cells, and the overall resonant frequency 
of the system can be temperature-tuned once balanced, $\pi$ mode critical coupling is achieved.

\subsection{UV Photocathode Laser}

The laser system used to illuminate the photocathode is a commercial chirped-pulse amplification (CPA) system, consisting of an oscillator, a grating stretcher, a regenerative amplifier, a grating compressor, and a frequency tripler. The system is based entirely on diode pumped solid-state laser (DPSSL) technology, which provides the stability needed to illuminate the photocathode reliably. The oscillator is a Titanium:Sapphire ( $\mathrm{Ti}_{\mathrm{Al}} \mathrm{Al}_{2} \mathrm{O}_{3}$ )-based FemtoSource PRO commercial system from Femtolasers Produktions $\mathrm{GmbH}$, capable of producing pulses as short as 10 fs via Kerr-lens mode-locking.

This oscillator is pumped by a $5 \mathrm{~W} \mathrm{CW}, 532 \mathrm{~nm}$ beam produced by the intracavity frequency-doubled, diodepumped, Nd: $\mathrm{YVO}_{4}$ Millennia V Laser from SpectraPhysics. Since 10 fs pulses are not needed for this application, are difficult to create and maintain, and will be unavoidably lengthened in this particular amplifier, the oscillator is operated without the final compensation optics, yielding a slightly chirped pulse that is $15 \mathrm{fs}$ in duration, with a bandwidth of $>70 \mathrm{~nm}$ centered around $800 \mathrm{~nm}$.

The oscillator was assembled with the output coupler mounted on a translation stage; using this set-up, the repetition rate of the oscillator can easily be adjusted between $79.120 \mathrm{MHz}$ and $79.155 \mathrm{MHz}$ without interfering with the Kerr-lens mode-locking process. In order to properly synchronize the laser with the rf in the gun, the oscillator cavity length was adjusted to produce a repetition rate of $79.138 \mathrm{MHz}$ (the $108^{\text {th }}$ subharmonic of the optimal gun operating frequency of $8.457 \mathrm{GHz}$ ).

The output of the oscillator is then coupled into a sub$50 \mathrm{fs}, 1 \mathrm{kHz}$ Spitfire system from Positive Light, which includes the grating stretcher, $\mathrm{Ti}: \mathrm{Al}_{2} \mathrm{O}_{3}$ regenerative amplifier, and grating compressor. The regenerative amplifier crystal is pumped by a $7 \mathrm{~W}$-average power, 1 $\mathrm{kHz}$, Q-switched, diode-pumped Nd:YLF Evolution Laser from Positive Light. This system, as currently configured, selects a pulse from the $79.138 \mathrm{MHz}$ pulse train and amplifies it to an energy of $0.4 \mathrm{~mJ}$, with a duration of 60 fs and an overall repetition rate of $1 \mathrm{kHz}$.

The amplified pulse is then directed into a frequency tripler produced by Spectra-Physics. This specific tripler design uses two $\beta$-Barium Borate (BBO) crystals for second- and third-harmonic generation. This converts the incoming IR beam to $266 \mathrm{~nm}$, which corresponds to the work function of copper at low fields. Due to fluctuations in the duration of the output pulse of the Spitfire, the intensity of the UV pulse varied between 5 and $7 \mu \mathrm{J}$ on a time scale of a few seconds. These fluctuations seem to be related to instabilities, e.g. from air currents, in the compressor section of the Spitfire. The UV beam is then injected onto the photocathode at 3 degrees from normal incidence using a custom-made high-vacuum "Y" piece attached to the output face of the gun.

\section{PHOTOELECTRON BEAM CHARACTERIZATION}

The first measurements of the photoelectron beam were made using a Faraday cup matched into $50 \mathrm{ohms}$. Taking the signal from the Faraday cup, the area under the signal voltage curve and Ohm's law can be used to determine the total charge in the beam. This particular measurement yields a total charge of $25 \mathrm{pC}$. Given the input energy of the UV laser as $6 \mu \mathrm{J}$, this yields a quantum efficiency for copper of $2 \times 10^{-5}$. The aforementioned shot-to-shot variations occurring at $20 \mathrm{~Hz}$ can be partially eliminated by averaging over several shots; good results were obtained by averaging for $5 \mathrm{~s}$ over 100 shots.

To measure the energy and energy spread of the electron beam, an electron spectrometer is used. A field of $0.73 \mathrm{kG}$ ( 3 A current) was used and the image produced by the deflected electrons on the phosphor screen was recorded on Polaroid film, which was then scanned at 600 dpi to make quantitative measurements. The energy of the beam was determined from the location of the image of the entrance slit to be $1.47 \mathrm{MeV}$, and the width of a spot gave a relative energy spread of $\Delta \gamma / \gamma_{0}<1.8 \%$ FWHM. We find that the 4-pixel FWHM spectrum has a momentum spread $\Delta u=0.068$, which corresponds to a relative energy spread $\Delta \gamma / \gamma=1.8 \%$.

To measure the beam emittance, direct examination of the beam divergence is made. First, a phosphor screen was used to determine the size of the electron beam, which was found to be $1.68 \mathrm{~mm}$ in diameter. This screen is then replaced by a thin tantalum (Ta) plate, with three holes of diameter $0.25,0.5$, and $1 \mathrm{~mm}$. Placing this plate in the beam path will completely stop the beam. The 1 mm-hole is centered on the beam to allow a small sample of the electron beam, with known diameter, to pass. The phosphor screen is placed 0.997 meter away from the Ta plate. The size of the electron beam at the phosphor is measured to be $1.84 \mathrm{~mm}$. Because the spot started with a diameter of $1 \mathrm{~mm}$ from the hole in the Ta, this means the edge divergence of the beam is $0.84 \mathrm{mrad}$, while the maximum core divergence, for on-axis electrons, is 1.84 mrad. Since the charge of this bunch is sufficiently small, space-charge effects on the emittance are negligible, and this divergence is a direct reflection of the transverse momentum spread of the beam. The transverse phase space of the beam can then be mapped by moving the Ta aperture and measuring the corresponding divergence angles. Using this technique, and limiting the count to $90 \%$ of the bunch charge, the normalized emittance of the beam is found to be $\varepsilon_{n} \approx 1.65 \pi \mathrm{mm} \mathrm{mrad}$. This is an excellent number, especially in view of the relatively low beam energy and absence of emittance compensation.

To get an estimate of the bunch length, a streak camera operating at its maximum streak speed of $20 \mathrm{ps} / \mathrm{mm}$ is used to image the phosphor screen. The streak image is 
captured on Polaroid film, which is scanned. When the electron beam is present, a prompt emission is observed on the streak image, followed by an image of the slow fluorescence of the phosphor. It is unclear if the observed line on the streak camera is purely Cherenkov radiation, or also produced by fast $\mathrm{x}$-rays interacting with the phosphor screen, but the duration of the light pulse in either case is directly correlated to the duration of the electron bunch, where we find it to be equal to the $2 \mathrm{ps}$ resolution of the camera itself.

We have performed experiments where the bunches propagate through a corrugated waveguide and couple to the slow waves supported by that structure. To demonstrate that the radiation produced is, indeed, coherent, we have measured the power radiated as a function of the bunch charge. The first experimental measurements consisted of measuring the $\mathrm{rf}$ power radiated by the bunches with a coupling horn, a waveguide section, and various detectors. We were able to confirm the emission of extremely short pulses of $\mathrm{rf}$ power up to Ka-band; at higher frequencies, the detector speed is believed to be insufficient. At low frequency, between X-band and Ka-band, we were able to measure pulses with a FWHM of 500 ps; this is to be compared with the detector rise-time, specified at $<600$ ps. At higher frequencies, the detectors are not fast enough to follow the pulses. The quadratic scaling of the power radiated versus the bunch charge is very clearly observed.

\section{CONCLUSIONS}

An X-band (8.574 GHz) rf photoinjector has been successfully operated, creating a reproducible $1.5-2 \mathrm{MeV}$ electron beam with low (1.8\%) energy spread, good (1.65 $\pi \mathrm{mm}$ mrad) emittance, and short ( $<2 \mathrm{ps})$ photoelectron bunches. Coherent synchrotron radiation was seen to be emitted by the electron beam as it propagated along the beamline; to our knowledge, this represents the first experimental study of coherent synchrotron radiation in a high-frequency photoinjector. The instabilities observed in the beam demonstrate the need for very careful control of laser pointing, energy, and pulse-length stability to produce a reliable high-quality beam.

\section{ACKNOWLEDGEMENTS}

This work was performed under the auspices of the U.S. Department of Energy by the University of California, Lawrence Livermore National Laboratory, partially through the Institute for Laser Science and Applications, under contract No. W-7405-Eng-48. Additional funding was provided by AFOSR Grant No. F4962099-1-0297 and NIH Contract No. N01-CO-97113.

\section{REFERENCES}

[1] Y. J. Chen, Nucl. Instrum. Methods Phys. Res., Sect. A 279, p. 433 (1989).

[2] J. Rosenzweig and E. Colby in Advanced Accelerator Concepts, 1994, edited by P. Schoessow, (AIP Conf. Proc. 335, Woodbury, NY, USA, 1995), p. 724.

[3] D. J. Gibson, F. V. Hartemann, et. al, Phys. Rev. Special Topics: Accel. and Beams. (In Review) 